Tohoku J. exp. Med., 1978, 125, 77-83

\title{
Study on the Porcine Tubular Basement Membrane
}

\author{
Hiroshi Munakata, Tokutaro Sato and Zensaku Yosizawa \\ Department of Biochemistry, Tohoku University School of \\ Medicine, Sendai 980
}

\begin{abstract}
Munakata, H., Sato, T. and Yosizawa, Z. Study on the Porcine Tubular Basement Membrane. Tohoku J. exp. Med., 1978, 125 (1), 77-83 — Porcine kidney slices were gently homogenized with $0.9 \% \mathrm{NaCl}$ in a Waring blender, followed by sieving of the homogenate to isolate tubules. The tubular basement membrane (TBM) was then prepared from the tubules by sonication in $1.0 \mathrm{M}$ $\mathrm{NaCl}$. Electron micrographs indicated a high degree of purity of the TBM with negligible contamination of fibrillar collagen. Analytical data showed that the TBM was a glycoprotein containing approximately $10 \%$ of carbohydrates. Of the sugar components, glucose and galactose comprised approximately $70 \%$ of the total carbohydrates and glycosylgalactosylhydroxylysine was detected in an alkaline hydrolyzate of the TBM. Besides them, mannose, fucose, glucosamine, galactosamine and sialic acid were also detected. The protein portion of the TBM contained hydroxyproline, hydroxylysine, half cystine, methionine and a large amount of glycine as the characteristic amino acids. Polyacrylamide gelelectrophoresis of the TBM gave 15 bands. __ tubular basement membrane; glucosylgalactosylhydroxylysine
\end{abstract}

The renal glomerular basement membrane (GBM) and tubular basement membrane (TBM) have been shown to be glycoprotein containing approximately $10 \%$ of carbohydrates (Kefalides and Winzler 1966; Spiro 1967a; Mahieu and Winand 1970; Westberg and Michael 1973; Ferwerda et al. 1974a, b; Sato et al. 1975a, b; Ferwerda and van Dijek 1975). As reviewed by Spiro (1972), GBM appeared to play a vital physiological role by acting as a selective filter, while TBM may play an important role in the highly selective reabsorption function of the tubule. Elucidation of their chemical changes under different pathological states would therefore give a clue to explain pathogenesis of certain diseases. Histologically, GBM is located between endothelium and podocytes, and continuous to the basement-membrane-like substance in mesangium, which would be mixed in GBM preparation. On the other hand, TBM is histologically simpler and easier to be isolated in pure state than GBM. It may therefore show a more precise figure of the chemical changes under the pathological conditions.

Although GBM has been studied extensively (Kefalides and Winzler 1966; Spiro 1967a; Mahieu and Winand 1970; Westberg and Michael 1973), there has been few reports concerning TBM (Mahieu and Winand 1970; Ferwerda et al.

Received for publication, October 4, 1977. 
1947a, b; Spiro 1972). Mahieu and Winand (1970) obtained TBM from human kidney. However, electronmicrogram and amino acid analysis indicated that their preparation was contaminated with a significant amount of fibrillar collagen. We isolated previously GBM from cortex and TBM from medulla of human kidney in a high degree of purity (Sato et al. 1975a, b). Subsequently, we prepared highly purified TBM by a simple method from porcine kidney without prior separation of cortex and medulla. This paper reports isolation and chemical compositions of the porcine TBM.

\section{Materials and Methods}

Materials. Porcine kidneys were obtained immediately after sacrifice of the animals from local slaughter-house and frozen in solid $\mathrm{CO}_{2}$. Other materials were from commerical sources.

Preparation of tubules. The kidney were eut into slices of about 7-8 $\mathrm{mm}$ in thickness and then homogenized gently with $0.9 \% \mathrm{NaCl}$ in a Waring blender for 5 min under cooling in an ice-bath. The homogenate was poured onto 80 mesh stainless steel sieve. The filtrate was discarded, and the residues on the sieve were re-homogenized by the same procedure. The resulting homogenate was again poured onto 80 mesh sieves, and the filtrate was passed through 115, 150, 200 and 250 mesh sieves, in succession. Phase-contrast microscopic examination showed that tubules retained on 250 mesh sieve. Therefore, the materials on this sieve were washed with ice-chilled $0.9 \% \mathrm{NaCl}$ until the tubules were freed from small glomerular fragments. Thereafter, the purified tubules were gently transferred into a centrifuge tube and then suspended in $0.9 \% \mathrm{NaCl}$. The suspension was centrifuged at $1,500 \mathrm{rpm}$ for $10 \mathrm{~min}$, and the sediments were frozen.

Preparation of $T B M$. The above sediments were suspended in $1.0 \mathrm{M} \mathrm{NaCl}$ and sonicated in a Kubota Insonator type $200 \mathrm{M}$ at 140 watt for 20 min under cooling in an ice-bath. Subsequently, each mixture was passed through 325 mesh sieve, and the filtrate was centrifuged at $3,000 \mathrm{rpm}$ for $10 \mathrm{~min}$. The resulting sediments were washed five times with ice-chilled 1.0 $\mathrm{M} \mathrm{NaCl}$, and then five times with ice-chilled distilled water, in succession. The sediments were suspended in water and then a portion was used for electron microscopic examination, and the remainings were lyophilized.

Electron microscopic examination. A portion of the above TBM suspension was fixed successively with glutaraldehyde and osmium tetroxide, and then embedded in Epon, followed by staining with uranyl acetate and lead citrate. Electron microscopic examination was generously carried out by Dr. S. Aizu in a Hitachi HS7S electron microscope.

Determination of constituents. A portion $(7 \mathrm{mg})$ of TBM was hydrolyzed with $1 \mathrm{ml}$ of $2 \mathrm{M} \mathrm{HCl}$ at $100^{\circ}$ for $6 \mathrm{hr}$ in a sealed tube. The hydrolyzate was passed through a coupled columns of Dowex $50 \times 4\left(200-400\right.$ mesh, $\mathrm{H}^{+}$form, $\left.1 \times 4 \mathrm{~cm}\right)$ and Dowex $1 \times 8(200-400$ mesh, formate form, $1.4 \times 4 \mathrm{~cm}$ ). The columns were washed with water. The column of Dowex 50 was then washed with $2 \mathrm{M} \mathrm{HCl}$. The water effluent was then lyophilized. Hexose content in the lyophilized sample was determined by the anthrone- $\mathrm{H}_{2} \mathrm{SO}_{4}$ method (Roe 1955). In addition, glucose, galactose and mannose were determined by gas chromatography. Gas chromatography of the trimethylsilyl derivatives of the methylglycosides was carried out in a Shimadzu GC-4B gas chromatograph according to the method of Yamakawa and Ueta (1964), using a $2-\mathrm{m}$ column packed with $3.3 \%$ SE-30 on Chromosorb $\mathrm{W}$ at $155^{\circ}$. The content of hexosamine in the eluate with $2 \mathrm{M} \mathrm{HCl}$ was determined by the method of Boas (1953). Determination of glucosamine and galactosamine was also carried out in a Hitachi 034 liquid chromatograph. Sialic acid was estimated by the method of Warren (1959) after hydrolysis with $0.05 \mathrm{M} \mathrm{H}_{2} \mathrm{SO}_{4}$ at $80^{\circ}$ for $1 \mathrm{hr}$.

Three mg of TBM was hydrolyzed in an ampule with $2 \mathrm{ml}$ of constant boiling $\mathrm{HCl}$ at 
$105^{\circ}$ for $24 \mathrm{hr}$ under nitrogen atmosphare, and $\mathrm{HCl}$ was removed by repeated evaporation in vacuo. The resulting residues were analyzed for amino acids in a Hitachi 034 liquid chromatography, using norleucine as an internal standard. Two $\mathrm{mg}$ of TBM were hydrolzyed with $0.2 \mathrm{ml}$ of $2 \mathrm{M} \mathrm{NaOH}$ at $105^{\circ}$ for $24 \mathrm{hr}$, and the hydrolyzate was neutralized to $\mathrm{pH}$ 4.5. The hydrolyzate was then analyzed for glucosylgalactosylhydroxylysine using $0.1 \mathrm{M}$ and $0.4 \mathrm{M}$ sodium citrate buffer $(\mathrm{pH} 5.0$ ) in a Hitachi 034 liquid chromatograph.

Three mg of TBM were extracted with 2-propanol-chloroform (11:7, v/v) overnight at room temperature by the procedure of Rose and Oklander (1965). The extract was evaporated to dryness in vacuo under nitrogen. The residue was then dissolved in chloroform-methanol $(1: 1, v / v)$, and the solution was washed by the method of Folch et al. (1957). The content of phospholipids in the extract was determined as phosphorus by the method of Bartilett (1959).

Polyacrylamide gel-electrophoresis. Sodium dodecyl sulfate (SDS) polyacrylamide gelelectrophoresis was carried out by the method of Hudson and Spiro (1972). Samples were incubated in $0.1 \mathrm{M}$ phosphate buffer ( $\mathrm{pH} 7.0$ ) containing $1 \% 2$-mercaptoethanol and $1 \%$ SDS at $37^{\circ}$ for $3 \mathrm{hr}$. Gel-electrophoresis was carried out at $8 \mathrm{~mA}$ per tube $(0.7 \times 9.0 \mathrm{~cm})$ for $5 \mathrm{hr}$, using $5 \%$ polyacrylamide. The gel was fixed with $10 \%$ trichloracetic acid, and then stained with Coomassie blue $(0.25 \%$ in $7.5 \%$ acetic acid).

\section{Results}

\section{Preparstion of $T B M$}

The purity of the tubules used for the preparation of TBM was more than $98 \%$ as judged by phase-contrast microscopic examination. Electron micrograph (Fig. 1) shows that TBM is amorphous and freed from subcellular contaminant and collagenous fiber.

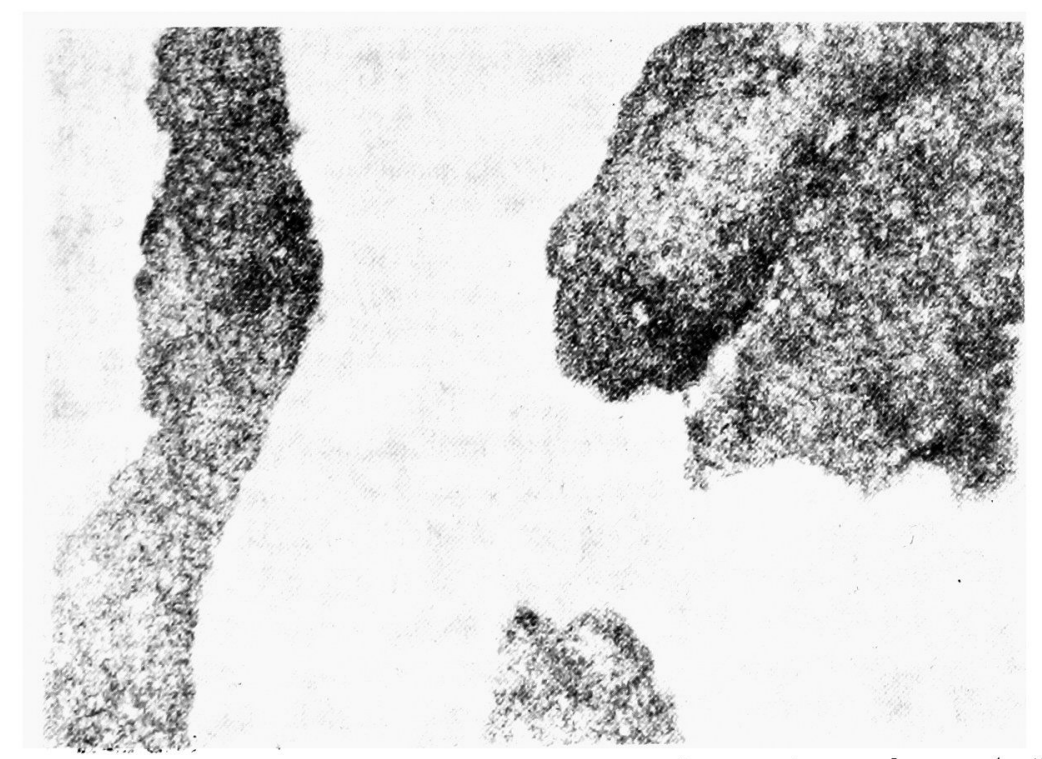

Fig. 1. Electron micrograph of porcine tubular basement membrane $(\times 50,000)$. Sample was fixed with glutarladehyde and $\mathrm{OsO}_{4}$ and embedded in Epon. Thin section was stained with uranyl acetate and lead citrate as described in the text. 
TABLE 1. Amino acid composition* of porcine tubular basement membrane

\begin{tabular}{lrr} 
membrane & & \\
\hline Hydroxyproline & 70.7 & $(6.23)$ \\
Aspartic acid & 67.2 & $(6.20)$ \\
Threonine & 39.2 & $(3.13)$ \\
Serine & 56.0 & $(3.95)$ \\
Glutamic acid & 94.5 & $(9.34)$ \\
Proline & 69.4 & $(5.37)$ \\
Glycine & 189.0 & $(9.53)$ \\
Alanine & 57.6 & $(3.45)$ \\
Half-cystine & 23.6 & $(1.91)$ \\
Valine & 39.0 & $(3.07)$ \\
Methionine & 14.4 & $(1.45)$ \\
Isoleucine & 29.2 & $(2.57)$ \\
Leucine & 62.4 & $(5.50)$ \\
Tyrosine & 25.9 & $(3.15)$ \\
Phenylalanine & 62.4 & $(6.92)$ \\
Hydroxylysine & 29.7 & $(3.24)$ \\
Lysine & 29.0 & $(2.85)$ \\
Histidine & 15.2 & $(1.58)$ \\
Arginine & 49.4 & $(5.78)$ \\
\hline
\end{tabular}

* Expressed as number of residues/1000 amino acid residues. (Data in parentheses expressed as $\mathrm{mg} / 100$ mg membrane)

TABLE 2. Carbohydrate composition* of porcine tubular basement membrane

\begin{tabular}{ll}
\hline Glucose & 3.14 \\
Galactose & 3.78 \\
Mannose & 0.83 \\
Fucose & 0.34 \\
Glucosamine & 1.35 \\
Galactosamine & 0.20 \\
Sialic acid $\dagger$ & 0.33 \\
\hline
\end{tabular}

* Expressed as $\mathrm{mg} / 100 \mathrm{mg}$ membrane.

$\dagger$ As $\mathrm{N}$-acetylneuraminic acid.

Contents of amino acids, carbohydrates and phospholipids

Analytical data of amino acids and carbohydrates of the porcine TBM are shown in Tables 1 and 2. As can be seen in Table 1, the TBM had similar amino acid composition to that of other basement membranes (Spiro 1967a; Mahieu and Winand 1970; Westberg and Michael 1973; Sato et al. 1975a, b), having hydroxylysine, hydroxyproline and a large amount of glycine as the characteristic amino acids. Sulfur-containing amino acids such as cysteine and methionine were also present in the TBM. The TBM contained approximately $10 \%$ of carbohydrates (Table 2). Of the sugar components, glucose and galactose comprised approximately $70 \%$ of the total carbohydrates. In addition, mannose, fucose, glucosamine, galactosamine and sialic acid were found. The molar ratio of glucosamine to galactosamine was $87: 13$.

Alkaline hydrolyzate of the TBM contained glucosylgalactosylhydroxylysine as 


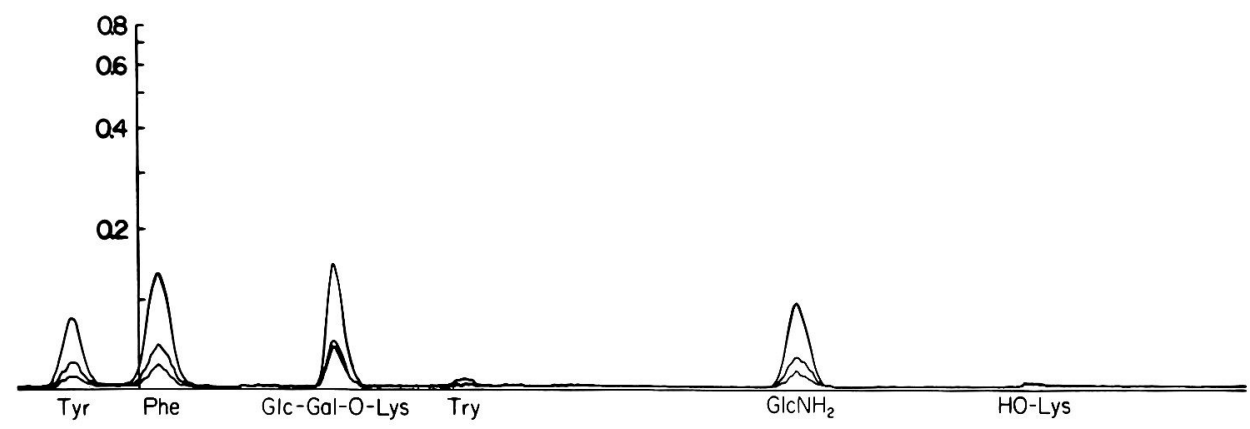

Fig. 2. Profile of glucosylgalactosylhydroxylysine (Glc-Gal-O-Lys) of porcine tubular basement membrane obtained by the amino acid analyzer (liquid chromatograph). Tyr, tyrosine; Phe, phenylalanine; Try, tryptophane; $\mathrm{GlcNH}_{2}$, glucosamine; HOLys, hydroxylysine.

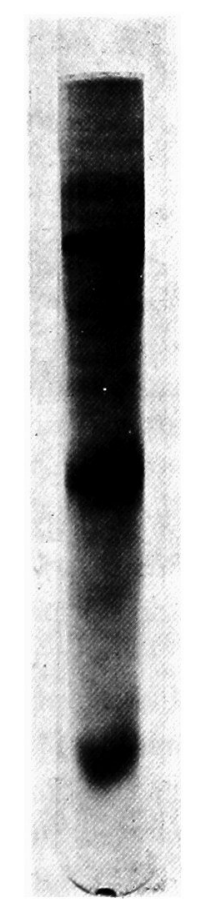

Fig. 3. Profile of polyacrylamide gelelectrophoretogram of porcine tubular basement membrane.

shown in Fig. 2.

The contents of phospholipid phosphorus of the TBM was $0.12 \mu \mathrm{g} / \mathrm{mg}$ of membrane, which was smaller than those reported previously (Spiro 1967a; Mahieu and Winand 1970; Westberg and Michael 1973; Sato et al. 1975a, b).

\section{Polyacrylamide gel-electrophoresis}

Polyacrylamide gel-electrophoresis of the porcine TBM gave 15 bands (Fig. 3). The pattern resembles that of GBM reported previously (Sato et al. 1975a). 


\section{Drscussion}

The human TBM was isolated by Mahieu and Winand (1970), using sieves and Potter-Elvehjem homogenizer. Their preparation was, however, contaminated electron microscopically with collagen fiber. Ferwerda et al. (1974b) isolated bovine TBM after disruption of the tissue by sieves. Although the purity of their tubules used for the preparation of TBM was more than $97 \%$, the procedure was troublesome. In the previous reports (Sato et al. 1975a, b), we prepared highly purified human tubules by strong homogenization with saline in a Waring blender from medulla. However, medulla of porcine kidney cannot be separated macroscopically from cortex. The kidney slices were therefore homogenized gently in a Waring blender to separate glomeruli and tubules. The present procedures for isolation of the tubules are simpler than those reported by other investigators (Mahieu and Winand 1970; Ferwerda et al. 1974b). Electron microscopic observation of the porcine TBM preparation indicated that contamination of fibrillar collagen in the TBM preparation was negligible. Contents of glycine and hydroxyproline in the TBM were smaller than those of collagen and content of hydroxylysine was rather high, which also indicated that contamination of collagen was very small.

The porcine TBM had similar compositions of amino acids and carbohydrates to those of other TBM and GBM (Spiro 1967a; Mahieu and Winand 1970; Westberg and Michael 1973; Ferwerda et al. 1974b; Sato et al. 1975a, b). However, the content of hydroxylysine of the porcine TBM was greater than that of the porcine GBM (15.8 residues/1000 amino acid residues, Munakata et al., unpublished data), as observed between human TBM and GBM (Sato et al. 1975a).

The presence of glucosylgalactosylhydroxylysine in the porcine TBM was revealed by the alkaline hydrolysis, as reported previously on human TBM and GBM (Sato et al. 1975a) and bovine TBM (Ferwerda and van Dijk 1975). Analytical data indicated also that the porcine TBM contained mannose, fucose, hexosamine and sialic acid, as reported for bovine TBM (Ferwerda and van Dijk 1975) and GBM (Spiro 1967b) and human TBM and GBM (Sato et al. 1975a).

According to Westberg and Michael (1973), the contents of phospholipid is smaller in the purer preparation of GBM. Phospholipid in the porcine TBM was $0.12 \mu \mathrm{g} / \mathrm{mg}$ of membrane, which was smaller than those reported previously (Spiro 1967a; Mahieu and Winand 1970; Westberg and Michael 1973; Sato et al. 1975a, b), suggesting high purity of the present preparation.

\section{Acknowledgment}

We thank Dr. S. Aizu, Department of Anatomy, Tohoku University School of Medicine, for electron microscopic examination.

\section{References}

1) Bartilett, G.R. (1959) Phosphorus assay in column chromatography. J. biol. Chem., $234,466-468$. 
2) Boas, N.F. (1953) Method for the determination of hexosamines in tissues. J. biol. Chem., 204, 553-563.

3) Ferwerda, W., Feltkamp-Vroom, T.M. \& Smith, J.W. (1974a) Collagen and glycoprotein components derived from bovine tubular basement membrane: Chemical and immunological properties. Biochem. Soc. Trans., 2, 640-642.

4) Ferwerda, W., Meijer, J.F.M., van der Eijnden, D.H. \& van Dijk, W. (1974b) Epithelial basement membrane of bovine renal tubuli. Hoppe-Seyler's Z. physiol. Chem., 355, 976-984.

5) Ferwerda, W. \& van Dijk, W. (1975) Epithelial basement membrane of bovine renal tubuli. Hoppe-Seyler's Z. physiol. Chem., 356, $1671-1678$.

6) Folch, J., Less, M. \& Sloane Stanley, G.H. (1957) A simple method for the isolation and purification of total lipids from animal tissues. J. biol Chem., 226, 497-509.

7) Hudson, B.G. \& Spiro, R.G. (1972) Studies on the native and reduced alkylated renal glomerular basement membrane. J. biol Chem., 247, 4229-4238.

8) Kefalides, N.A. \& Winzler, R.J. (1966) The chemistry of glomerular basement membrane and its relation to collagen. Biochemistry, 5, 702-713.

9) Mahieu, P. \& Winand, R.J. (1970) Chemical structure of tubular and glomerular basement membrane of human kidney. Europ. $J$. Biochem., 12, 410-418.

10) Roe, J.H. (1955) The determination of sugar in blood and spinal fluid with anthrone reagent. J. biol Chem., 212, 335-343.

11) Rose, H.G. \& Oklander, M. (1965) Improved procedure for the extraction of lipids from human erythrocytes. J. Lipid Res., 6, 428-431.

12) Sato, T., Munakata, H., Yoshinaga, K., \& Yosizawa, Z. (1975̃a) Chemical composition of glomerular and tubular basement membrane of human kidney. Tohoku $J$. exp. Med., 115, 299-306.

13) Sato, T., Munakata, H., Yoshinaga, K. \& Yosizawa, Z. (1975b) Comparison of the chemical composition of glomerular and tubular basement membranes obtained from human kidneys of diabetics and non-diabetics. Clin. chim. Acta, 61, 145-150.

14) Spiro, R.G. (1967a) Studies on the renal glomerular basement membrane. J. biol Chem., 242, 1915-1922.

15) Spiro, R.G. (1967b) Studies on the renal glomerular basement membrane. J. biol. Chem., 242, 1923-1932.

16) Spiro, R.G. (1972) Basement membrane and collagens. In: Glycoproteins, edited by A. Grottschalk, 2nd ed., Elsevier, Amsterdam, pp. 964-999.

17) Warren, L. (1959) The thiobarbituric acid assay of sialic acids. J. biol. Chem., 234, 1971-1975.

18) Westberg, N.G. \& Michael, A.F. (1973) Human glomerular basement membrane: Chemical composition in diabetes mellitus. Acta med. scand., 194, 39-47.

19) Yamakawa, T. \& Ueta, N. (1964) Gas-liquid chromatography of carbohydrates. Jap. J. exp. Med., 34, 37-51. 